\title{
Spontaneous splenic rupture in polyarteritis nodosa
}

\author{
G.A. Ford*, J.R. Bradley, D.S. Appleton, Sathia Thiru and R.Y. Calne
}

Addenbrooke's Hospital, Hill's Road, Cambridge CB2 2QQ, UK.

\begin{abstract}
Summary: A 28 year old man presented with haematuria and renal failure. Renal arteriography revealed bleeding into the pelvis of a solitary right kidney from a hilar artery. Multiple aneurysms were noted in the splenic artery. The spleen subsequently ruptured spontaneously and was removed at laparotomy together with the right kidney. Histological examination of the spleen and kidney revealed an acute necrotizing vasculitis involving medium-sized and smaller arteries confirming a diagnosis of polyarteritis nodosa. An area of infarction involved a subcapsular area of the spleen. Spontaneous splenic rupture is a rare but important complication of systemic vasculitides.
\end{abstract}

\section{Introduction}

Splenic rupture has been reported as a rare complication of systemic vasculitides. We report a case in a patient with histologically confirmed polyarteritis nodosa involving predominantly the spleen and a solitary kidney.

\section{Case report}

A 28 year old man developed right loin pain and haematuria. He gave a history of malaise, anorexia, weight loss and muscle aches over the previous 3 months. On admission to hospital blood pressure was $100 / 50 \mathrm{mmHg}$ and pulse $70 /$ minute. The right kidney was tender and enlarged and he was passing fresh blood with clots per urethra. Haemoglobin was $10.2 \mathrm{~g} /$ $\mathrm{dl}$, white cell count $14.8 \times 10^{9} / 1$, platelet count $345 \times 10^{9} / 1$ and erythrocyte sedimentation rate $83 \mathrm{~mm} / \mathrm{h}$. Blood urea was $23.1 \mathrm{mmol} / \mathrm{l}$ and creatinine $795 \mu \mathrm{mol} / 1$. Following admission haematuria persisted with further deterioration in his renal function and he required resuscitation with blood transfusion and haemodialysis. Abdominal computed tomographic scan revealed an absent left kidney and an enlarged right kidney with a mass in the upper pole. Aortogram and selective renal angiograms showed a jet of contrast passing directly from a hilar artery to the renal pelvis, and stretching and irregularity of intra-renal vessels with poor forward flow (Figure 1). There were no convincing renal artery aneurysms but multiple aneurysms were present in the splenic artery. Follow-

Correspondence: J.R. Bradley, B.Med.Sci., M.R.C.P.

*Present address: Gloucester Royal Hospital, Great Western Road, Gloucester GL1 3NN, UK.

Accepted: 12 May 1986 ing angiography he developed severe abdominal pain and was found to be hypotensive with an abdominal fluid thrill. At laparotomy haemoperitoneum secondary to a ruptured spleen was found. The right kidney was enlarged and haemorrhagic with a tense cystic swelling in the upper pole. The lower pole was

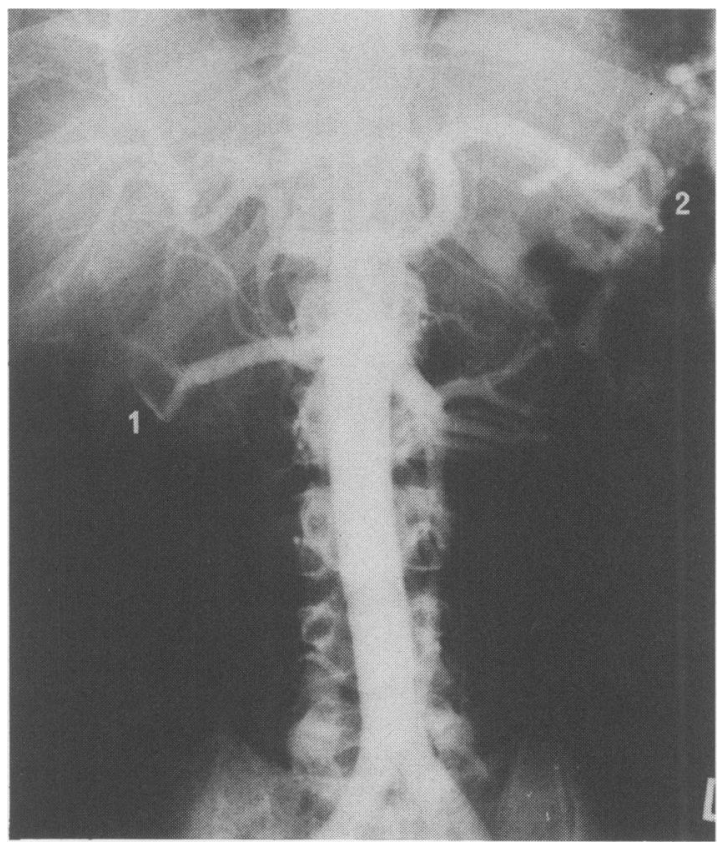

Figure 1 Aortogram and selective renal angiogram showing (1) the jet of contrast passing directly from a hilar artery into the renal pelvis, and (2) the multiple splenic artery aneurysms.

(C) The Fellowship of Postgraduate Medicine, 1986 
infarcted. The spleen and kidney were both removed.

Examination of the spleen revealed a triangular area of infarction of approximately $5 \mathrm{~cm}$ in diameter extending from subcapsule to the hilum. Histology revealed evidence of an acute fibrinoid vasculitis with destruction of the elastic lamina and media in the blood vessels near and in the infarcted area. The kidney had areas of recent infarction with extensive haemorrhage. An acute necrotizing vasculitis involved both mediumsized and smaller arteries within the renal parenchyma, and a branch of the renal artery in the hilum showed focal total necrosis of the wall together with a surrounding haematoma filling the pelvis. The histological appearances were diagnostic of polyarteritis nodosa.

Post-operatively he was maintained on haemodialysis and started on prednisolone $60 \mathrm{mg} / \mathrm{day}$. He made a good recovery and has remained well over the last year on haemodialysis and a reducing dose of steroids.

\section{Discussion}

Acute abdominal complications of polyarteritis nodosa have been described previously (Zizic et al.,

\section{References}

FALLINGBORG, J., LAUSTEN, J., JAKOBSEN, J., WINTHER, P. \& SVANHOLM, H. (1985). Atraumatic rupture of the spleen in periarteritis nodosa. Acta Chirurgica Scandinavica, 151, 85 .

HUTTUNEN, R., SEPPALA, A., MOKKA, R. \& LARMI, T.K.I. (1975). Spontaneous rupture of the liver and spleen: Report of a successfully treated case with immunopathological reaction. Surgery, 77, 722.
1982), the most common syndrome requiring laparotomy being mesenteric arteritis with infarcted bowel and peritonitis. Spontaneous splenic rupture has been reported as a rare complication of systemic lupus erythematosus (Krauser, 1976), in one case in which evidence of an acute immunological reaction was found on histological examination of the spleen but a firm diagnosis was not established (Huttunen $e t$ al., 1975) and in one other case of histologically confirmed polyarteritis nodosa (Fallingborg et al., 1985). The mechanism involved is unclear. In the case described previously rupture of a microaneurysm within the spleen was felt to have been the precipitating event. In the case we have described splenic rupture seems to have been the result of a vasculitis with infarction of a subcapsular area of the spleen. Although splenic rupture occurred following angiography it was felt unlikely that this could have been contributory. The procedure was limited to a free flush aortogram and selective right renal angiogram and the patient was haemodynamically stable throughout. Splenic rupture should be considered in patients with polyarteritis nodosa presenting with an acute abdomen.
KRAUSER, R.E. (1976). Spontaneous rupture of the spleen in systemic lupus erythematosus. Journal of the American Medical Association, 236, 1149.

ZIZIC, T.M., CLASSEN, J.N. \& STEVENS, M.B. (1982). Acute abdominal complications of systemic lupus erythematosus and polyarteritis nodosa. American Journal of Medicine, 73, 525 . 